\title{
Infectious outcomes after splenectomy for trauma, splenectomy for disease and splenectomy with distal pancreatectomy
}

\author{
Leonel Camejo ${ }^{1} \cdot$ Nandini Nandeesha $^{1} \cdot$ Kevin Phan $^{1} \cdot$ Khattiya Chharath $^{1} \cdot \operatorname{Thanh}_{\operatorname{Tran}^{1}} \cdot$ David Ciesla $^{1}$. \\ Vic Velanovich ${ }^{1}[$ ]
}

Received: 20 September 2021 / Accepted: 15 January 2022 / Published online: 24 January 2022

(c) The Author(s), under exclusive licence to Springer-Verlag GmbH Germany, part of Springer Nature 2022

\begin{abstract}
Introduction The spleen provides a unique immune function in its production of opsins directed against encapsulated bacteria. Splenectomy, therefore, increases the risk of infections in patients as well as post-operative complications. This study aims to assess the risk of post-operative complications within 5 years of splenectomy by indication for splenectomy: trauma, disease, or in association with a distal pancreatectomy for pancreatic disease. The relationship between vaccination and infectious outcomes was also investigated.

Methods This study is a review of splenectomy cases between June 2005 and June 2015 at a single institution. Infection, splenectomy indication, and vaccination history were identified from electronic medical records and lab test confirmations. Data was analyzed using Student's $t$ test for continuous variables, the Mann-Whitney $U$ test for ordinal variables, and a Chi-square/Fisher exact test for categorical variables.

Results A total of 106 splenectomy patients were included: 35 traumatic (74\% male) and 71 non-traumatic causes (42\% male) with no significant difference in age. There were no statistical differences in complications during splenectomy and vaccination administration between the splenectomy indication groups: trauma, disease, and with distal pancreatectomy. There was a statistically significant higher infection rate within 5 years post-splenectomy in the non-traumatic vs traumatic group (42\% vs $14.0 \%, p=0.0040)$ with majority gastrointestinal $(7 / 38)$ and respiratory $(5 / 38)$ and surgical wound infections (3/38) observed in non-traumatic versus traumatic, respectively.

Conclusion Results from data analysis show a statistically significant difference in rates of infection within 5 years postoperatively between traumatic versus non-traumatic indications for splenectomies, with the non-traumatic group experiencing a higher rate of infectious outcomes. The non-traumatic group included patients with disease and distal pancreatectomy indications. This suggests that patients who have non-traumatic causes may be at a higher risk of developing infections following splenectomy procedure. Additionally, vaccinations did not appear to have a protective effect.
\end{abstract}

Keywords Splenectomy $\cdot$ Post-operative infection $\cdot$ Retrospective chart review

\section{Abbreviations}

TGH Tampa General Hospital

NIS Nationwide Inpatient Sample

APC Antigen-presenting cells

ITP Idiopathic thrombocytopenic purpura

OPSI Overwhelming post-splenectomy infection

Vic Velanovich

vvelanov@usf.edu

1 Department of Surgery, Division of Gastrointestinal Surgery, Morsani College of Medicine, University of South Florida, 5 Tampa General Circle, Suite 740, Tampa, FL 33606, USA

\section{Introduction}

Splenectomy is indicated for a variety of disease processes. For disease, these include a variety of benign and malignant hematologic disease [1], splenic cysts [1], as well as splenomegaly with and without associated hypersplenism [2]. For trauma, although a variety of splenic preservation techniques are available, splenectomy is still life-saving in severe injury [3]. Lastly, for anatomical reasons, the spleen is frequently resected en bloc with the distal pancreas for pancreatic disease involving the body and tail of the pancreas [4]. Splenectomy may be done through an open midline, left subcostal laparotomy incision, or laparoscopically $[5,6]$ which is associated with quality of life advantages [7]. 
While it is not an organ essential for life, the spleen, nevertheless, has a unique immune function. It provides antigenpresenting cells (APCs) to facilitate B lymphocyte responses against blood-borne antigens [8]. Its structure allows for the filtration of blood pathogens and abnormal cells. Patients without a spleen may lack immunity due to the absence of these unique APCs and have impaired filtration ability. As such, post-splenectomy patients are at risk for a variety of long-term adverse events [9], the most devastating being overwhelming post-splenectomy infection (OPSI) [10]. This is due to polysaccharide encapsulated bacteria, which are otherwise neutralized by spleen [11]. A review study by Sinwar reports an overall 5\% lifetime risk for OPSI in asplenic patients [10]. The mortality rate associated with this infection is reported to be between 38 and $70 \%$ on average, but can be as low as $10 \%$ [10]. In addition, asplenic patients are further at risk for other bacterial and viral infections as a result of immunosuppression [9].

Because of the deleterious effects of splenectomy, a variety of splenic preservation techniques have been development for splenic trauma [12], resection of splenic cysts [13], and distal pancreatectomy [14]. Nevertheless, despite these efforts, there may be no alternatives to a total splenectomy. Therefore, to address the immunological consequences of splenectomy, vaccination has become standard. Clinical guidelines for splenectomy recommend vaccinating patients against the $S$. pneumoniae, $N$. meningitidis, $H$. influenzae type $b$ and influenza virus at least two weeks prior to surgery in elective cases or two weeks post-operatively for emergent splenectomies [15].

Although splenectomy patients are preventatively vaccinated for OPSI, they are still at risk of developing nonOPSI infections. A systematic review by Rodeghiero and Ruggeri [16] found the risk of post-splenectomy infection was highest within the first 90 days after splenectomy, but persistent at a much lower rate even after one year. They also determined that the risk of infection was related to the indication for splenectomy, whether for disease or trauma. However, they did not assess splenectomy in association with distal pancreatectomy for pancreatic disease. Therefore, a better understanding of infection occurrence after the immediate post-operative period is needed. The primary aim of this study is to determine if there is any difference in infectious events within 5 years of the splenectomy by indication for splenectomy: trauma, disease, or in association with a distal pancreatectomy for pancreatic disease. Secondary aims are to assess variation in vaccine administration and to determine if this variation was associated with infectious outcomes.

\section{Methods}

This study was approved by the institutional review boards of the University of South Florida and Tampa General Hospital.

The electronic medical records of patients who underwent a splenectomy for trauma, disease, or in association with a distal pancreatectomy at Tampa General Hospital between June 1, 2005, and June 31, 2015, to allow for a minimum of 5 year follow were accessed. Inclusion criteria were splenectomy patients $\geq 18$ years old for any indication, whether planned or unplanned. Patients were excluded if they met any of the following criteria: $\leq 17$ years old; underwent splenectomy outside of the given timeframe; if there was any splenic preservation; subject was involved in ongoing medical litigation; or subject was incarcerated. The definition of splenectomy for trauma was any splenic injury caused by blunt or penetrating force, in addition to intra-operative or procedural-related iatrogenic injury. Splenectomy for disease was defined as any indication for the treatment of benign or malignant hematologic or lymphatic system disease. The definition of splenectomy in association with a distal pancreatectomy was any time the spleen was resected en bloc with the distal pancreas for benign or malignant disease, regardless of splenic involvement.

Data gleaned from the records were as follows: patient age, patient sex, name of the attending physician, diagnosis at admission, presenting symptoms, indication for splenectomy, grade of splenic injury for trauma as determined by computed tomographic scanning or intra-operative assessment, date of splenectomy, splenectomy approach (open vs laparoscopic), intra- and post-operative complications, length of stay, vaccination administration and timing, infection within five years, length of time between splenectomy and infection. After the data was collected on a limited access Excel document, it was transferred over to REDCap, a secure web application for building and managing research databases.

\section{Statistical analysis}

Data was analyzed using descriptive statistics, Student's $t$ test for normally distributed continuous variables (presented as means with standard deviations), the Mann-Whitney $U$ test for non-normally distributed variables (presented as medians with interquartile ranges), Fisher exact test for $2 \times 2$ categorical variables containing cells $<5$, and Chi-squared test for greater than $2 \times 2$ categorical variables. A $p$ value of $\leq 0.05$ was considered statistically significant. 


\section{Results}

\section{Demographics comparing trauma and non-trauma splenectomy groups}

A total of 106 patients underwent splenectomy in the defined timed period and met the inclusion criteria for the study. We had a 5-year follow-up on $79(75 \%)$ patients. Table 1 presents the data comparing patients undergoing splenectomy for trauma and non-trauma indications. For the trauma patients, $25(71 \%)$ were due to blunt or penetrating trauma, with the mechanisms of splenic injury including motor vehicle collision, automobile-pedestrian accidents, and physical assaults, while 10 (29\%) were iatrogenic. Table 2 presents the indications for non-trauma splenectomy and grade distribution of splenic injury. There was a higher frequency of male patients in the trauma category, compared to the non-trauma category (Table 1). There was no difference in age between the groups (Table 1). All of the trauma splenectomies were done via an open laparotomy, while there was an even distribution between laparoscopic and open operations in the non-trauma group. Overall length of stay was longer for the trauma group. Twelve different attending surgeons were involved in the operations of the trauma group, while 11 different surgeons were involved in the non-trauma group.

Table 1 Patient characteristics

\begin{tabular}{llll}
\hline Splenectomy indication: & $\begin{array}{l}\text { Trauma } \\
(n=35)\end{array}$ & $\begin{array}{l}\text { Non-trauma } \\
(n=71)\end{array}$ & $p$ value \\
\hline $\begin{array}{lll}\text { Age, mean } \pm \text { SD } \\
\text { range] }\end{array}$ & $\begin{array}{l}50 \pm 19 \\
{[18-89]}\end{array}$ & $\begin{array}{l}53 \pm 16 \\
{[19-80]}\end{array}$ & NS \\
$\begin{array}{l}\text { Gender } \\
\quad \text { Male }\end{array}$ & $26(74 \%)$ & $30(42 \%)$ & 0.0019 \\
$\quad$ Female & $9(26 \%)$ & $41(58 \%)$ & \\
Surgical approach & & & \\
$\quad$ Open & $35(100 \%)$ & $36(51 \%)$ & 0.0001 \\
$\quad$ Laparoscopic & & $35(49 \%)$ & \\
Length of stay, days, median with & $12(6-27)$ & $8(4-13)$ & 0.0039 \\
$\quad$ interquartile range & & & \\
Complications during procedure & $3(9 \%)$ & $5(7 \%)$ & NS \\
Complications following proce- & $16(46 \%)$ & $14(20 \%)$ & 0.0052 \\
$\quad$ dure & & & \\
Vaccinations administered: & & & \\
$\quad \begin{array}{l}\text { None } \\
\quad \text { Preoperatively }\end{array}$ & $7(20 \%)$ & $21(30 \%)$ & 0.0002 \\
$\quad \begin{array}{l}\text { Post-operatively } \\
\text { Unknown }\end{array}$ & $1(3 \%)$ & $25(35 \%)$ & \\
Infection within 5 years & $22(63 \%)$ & $20(28 \%)$ & \\
\hline & $5(14 \%)$ & $5(7 \%)$ & \\
\hline & $5(14 \%)$ & $30(42 \%)$ & 0.0040 \\
\hline
\end{tabular}

Table 2 Disease and injury distribution

\begin{tabular}{ll}
\hline Indication for non-trauma splenectomy & Grade of splenic Injury \\
\hline Splenectomy only ( $n=37):$ & I: $2(6 \%)$ \\
Splenomegaly/hypersplenism: $13(18 \%)$ & II: $5(14 \%)$ \\
Immune thrombocytopenic purpura: 12 & III: $7(20 \%)$ \\
$(17 \%)$ & IV: $9(28 \%)$ \\
Splenic vein thrombosis: $2(3 \%)$ & V: $12(34 \%)$ \\
Splenic abscess: $2(3 \%)$ & \\
Splenic infarction: $2(3 \%)$ & \\
Acute aortic dissection (splenic ischemia): & \\
$1(1 \%)$ & \\
Autoimmune hemolytic anemia: $1(1 \%)$ & \\
Splenic lymphoma: $2(1 \%)$ & \\
Splenic artery aneurysm: 1 (1\%) & \\
Spontaneous bleeding due to cirrhosis: $1 \%$ & \\
(1\%) & \\
Splenectomy with distal pancreatectomy & \\
$(n=34):$ & \\
Pancreatic neoplasm/mass: 28 (39\%) & \\
Chronic pancreatitis: 5 (7\%) & \\
Acute necrotizing pancreatitis: $1(1 \%)$ &
\end{tabular}

\section{Demographics comparing non-trauma splenectomy only and distal pancreatectomy/splenectomy groups}

Table 3 presents the demographic data between patients undergoing splenectomy for disease only compared to patients undergoing splenectomy with distal pancreatectomy for pancreatic disease. There was no difference in the sex distribution between groups, but patients in the distal pancreatectomy/splenectomy group were older and had a longer length of stay (Table 3). There were 7 different surgeons involved in the splenectomy only group and 8 different surgeons in the distal pancreatectomy/splenectomy group.

\section{Intra- and post-operative complications}

Tables 1 and 3 present the frequency of intra-operative and post-operative complications in the study groups. Most of the complications in the trauma group occurred post-operatively. In the splenectomy-only group, there were three intra-operative complications: bleeding from the splenic hilum and need for transfusion, injury to the spleen causing excessive bleeding and need for transfusion, as well as mesenteric ischemia in a patient with an acute aortic dissection. In the distal pancreatectomy/splenectomy group, there were 2 intra-operative complications: excessive bleeding from the splenic hilum requiring transfusion and injury to the superior mesenteric artery.

Post-operative complications were more common in the trauma group, compared to the non-traumatic group (Table 1). Complications were also more common in the pancreatectomy/splenectomy group compared to the splenectomy-only group (Table 3 ). In the traumatic group, 
Table 3 Comparison of nontrauma splenectomy only with non-trauma splenectomy with distal pancreatectomy

\begin{tabular}{|c|c|c|c|}
\hline & $\begin{array}{l}\text { Splenectomy only } \\
(n=37)\end{array}$ & $\begin{array}{l}\text { Splenectomy with distal } \\
\text { pancreatectomy } \\
(n=34)\end{array}$ & $p$ value \\
\hline $\begin{array}{l}\text { Age, mean } \pm S D \text {, years } \\
\text { [range] }\end{array}$ & $\begin{array}{l}47 \pm 16 \\
{[19-79]}\end{array}$ & $\begin{array}{l}59 \pm 12 \\
{[39-80]}\end{array}$ & 0.0056 \\
\hline \multicolumn{4}{|l|}{ Gender } \\
\hline Male $(\%)$ & $14(38 \%)$ & $16(47 \%)$ & \multirow[t]{2}{*}{ NS } \\
\hline Female $(\%)$ & $23(62 \%)$ & $18(53 \%)$ & \\
\hline \multicolumn{4}{|l|}{ Surgical approach } \\
\hline Open $(\%)$ & $17(46 \%)$ & $19(56 \%)$ & \multirow[t]{2}{*}{ NS } \\
\hline Laparoscopic (\%) & $20(54 \%)$ & $15(44 \%)$ & \\
\hline $\begin{array}{l}\text { Length of stay, days, median with inter- } \\
\text { quartile range }\end{array}$ & $5(3-9)$ & $11(6-17)$ & 0.0003 \\
\hline Complications during procedure $(\%)$ & $3(8 \%)$ & $2(6 \%)$ & NS \\
\hline Complications following procedure & $4(11 \%)$ & $10(29 \%)$ & 0.0491 \\
\hline \multicolumn{4}{|l|}{ Vaccinations administered: } \\
\hline None & $5(14 \%)$ & $16(47 \%)$ & \multirow[t]{4}{*}{$<0.0001$} \\
\hline Preoperatively & $22(59 \%)$ & $3(9 \%)$ & \\
\hline Post-operatively & $7(19 \%)$ & $13(38 \%)$ & \\
\hline Unknown & $3(8 \%)$ & $2(6 \%)$ & \\
\hline Infection within 5 years & $17(46 \%)$ & $13(38 \%)$ & NS \\
\hline
\end{tabular}

there were 6 deaths, 3 patients with leukocytosis, 2 surgical wound complications, and one of each of the following: deep venous thrombosis, acute respiratory failure, paraplegia (most likely related to the inciting trauma), and anastomotic leak. In the splenectomy-only group, post-operative complications included one of each of the following: death (due to an acute aortic dissection), pulmonary embolism, acute respiratory distress syndrome, and thrombocytopenia. In the distal pancreatectomy/splenectomy group, there were 2 of each of the following compilations: death and hospital-acquired pneumonia, empyema, atrial fibrillation, stroke, surgical wound complication, hypoglycemia, and hyperamylasemia.

\section{Vaccination administration}

Tables 1 and 3 present the vaccination administration data. There were significant differences in vaccine administration between the trauma and non-trauma groups and between the splenectomy-only and distal pancreatectomy/splenectomy groups.

\section{Post-splenectomy infections within 5 years}

Table 1 presents the post-splenectomy infection rate for trauma and non-trauma splenectomy. Of these infections, $30(86 \%)$ occurred after discharge, while $5(14 \%)$ prior to discharge. There was a statistically significant higher rate of infectious outcomes in the non-trauma compared to the trauma group. Table 3 presents the infection rates of the splenectomy-only and distal pancreatectomy/splenectomy groups. There was no difference in the rate of infectious occurrences between the groups. However, when compared to the trauma group, both the splenectomy only group ( $p=0.0036)$ and distal pancreatectomy/splenectomy group ( $p=0.0235$ ) had statistically significant higher rates of infectious outcomes. We also found that there was no difference in age between those without and those with an infectious outcome $(51 \pm 18$ years vs $52 \pm 14$ years, respectively, $p=\mathrm{NS}$ ), nor sex distribution (male $54 \%$ :female $46 \%$ vs male 51\%:female $49 \%$, respectively, $p=$ NS). Table 4

Table 4 Distribution of post-operative infections within 5 years

\begin{tabular}{lll}
\hline Infection & Non-trauma & Trauma \\
\hline Bacteremia/septicemia & $2(3 \%)$ & \\
Septic shock & $1(1 \%)$ & $1(3 \%)$ \\
Surgical site infection & $4(6 \%)$ & $2(6 \%)$ \\
Leukocytosis (presumed infection) & $3(4 \%)$ & $1(3 \%)$ \\
Pneumonia/respiratory & $4(6 \%)$ & \\
Urinary tract infection & $7(10 \%)$ & \\
Pyelonephritis & $1(1 \%)$ & \\
Intra-abdominal abscess & $3(4 \%)$ & \\
Pancreatitis & $1(1 \%)$ & \\
Cholecystitis & $1(1 \%)$ & \\
Clositrium difficile colitis & $1(1 \%)$ & \\
Human metapneumovirus & $1(1 \%)$ & \\
SARS CoV-2 & $1(1 \%)$ & \\
Osteomyelitis & & \\
\hline
\end{tabular}


Table 5 Distribution of microbial organisms

\begin{tabular}{ll}
\hline Organism & Distribution (\%) \\
\hline Gram positive organisms & \\
Staphylococcus aureus & $4(14 \%)$ \\
Staphylococcus epidermidis & $2(7 \%)$ \\
Streptococcus faecalis & $3(11 \%)$ \\
Streptococcus viridans & $3(11 \%)$ \\
Streptococcus pneumonia & $1(4 \%)$ \\
Streptococcus mitis & $1(4 \%)$ \\
Diphtheroid bacilli & $1(4 \%)$ \\
Clostridium difficile & $1(4 \%)$ \\
Gram negative organisms & \\
Escherichia coli & $4(14 \%)$ \\
Klebsiella pneumoniae & $2(7 \%)$ \\
Haemophilus influenza & $1(4 \%)$ \\
Prevotella buccae & $1(4 \%)$ \\
Psuedomonas aeruginosa & $1(4 \%)$ \\
Yeast & \\
Candida albicans & $2(7 \%)$ \\
Candida tropicalis & $1(4 \%)$ \\
\hline
\end{tabular}

shows the distribution of infectious occurrences. These included bacterial and viral infections distributed over a wide range of organ systems. Table 5 shows the distribution of microorganisms causing the infections, which include encapsulated and non-encapsulated bacteria as well as yeasts. The median time to infection was 4 months (range: 1 to 48 months).

Table 6 shows the occurrence of infectious outcomes by vaccination status. There were no statistically significant differences among the vaccination status groups overall or by splenectomy type. Table 7 shows the occurrence of infectious outcomes by splenic injury grade. All infectious outcomes occurred in the grades I to III group and this was a statistically higher rate than the grades VI and V group.

\section{Discussion}

Splenectomy is performed to treat a wide variety of conditions. The spleen is not required for life but still plays a role in blood filtration and immune response. Consequently, asplenic patients are more prone to a variety of adverse events [9]. The most feared infection that is unique to splenectomy patients is OPSI [17]. Patients without a spleen have a greater risk of death by approximately 200 -fold, from septicemia compared to those with a normally functioning spleen [18]. What we have shown is that infectious outcomes occur more often in patients undergoing splenectomy for disease or in conjunction with a distal pancreatectomy compared to splenectomy for trauma. There did not seem to be a particular pattern as to the types of infections, nor did vaccine administration or timing confer a protective effect.

Prima facie, the fact that vaccination administration did not seem to affect infectious outcomes is puzzling. However, this may simply be that the present vaccination regimen is designed for encapsulated organisms [19] and these infections may be from other microbes. This will require additional research.

It has generally been accepted that age and chronic disease has a more significant impact on developing an infection post-splenectomy. The effects of aging on the immune system can be profound. There is a decrease in the production of new adaptive immune cells such as lymphocytes, as well as an age-associated decline in certain subsets of protective $\mathrm{CD} 4+\mathrm{T}$ cells and B cells in the thymus gland or bone marrow [20,21]. Because of this, age was suspected to be a noteworthy risk factor in influencing
Table 6 Comparison of lateterm infections $(\leq 5$ years from splenectomy) by vaccination administration

Table 7 Comparison of lateterm infections $(\leq 5$ years from splenectomy) by splenic injury grade

\begin{tabular}{lllll}
\hline Vaccination administration & $\begin{array}{l}\text { Overall } \\
\text { \#infections/total }(\%)\end{array}$ & $\begin{array}{l}\text { Splenec- } \\
\text { tomy only } \\
\text { \#infec- } \\
\text { tions/total }\end{array}$ & $\begin{array}{l}\text { Splenectomy with } \\
\text { distal pancreatectomy } \\
\text { \#infections/total }\end{array}$ & $\begin{array}{l}\text { Trauma } \\
\text { \#infections/total }\end{array}$ \\
\hline None & $8 / 28(29 \%)$ & $2 / 5$ & $6 / 16$ & $0 / 7$ \\
Preoperatively & $12 / 26(46 \%)$ & $9 / 22$ & $2 / 3$ & $1 / 1$ \\
Post-operatively & $11 / 41(27 \%)$ & $5 / 7$ & $4 / 13$ & $2 / 22$ \\
Unknown & $4 / 11(36 \%)$ & $1 / 3$ & $1 / 2$ & $2 / 5$ \\
$p$ value & $\mathrm{NS}$ & $\mathrm{NS}$ & $\mathrm{NS}$ & $\mathrm{NS}$ \\
\hline
\end{tabular}

\begin{tabular}{lllllll}
\hline & I & II & III & IV & V & Unknown \\
\hline Infection/total & $0 / 2$ & $1 / 5$ & $2 / 5$ & $0 / 9$ & $0 / 11$ & $1 / 2$ \\
Infection/total & $3 / 12(25 \%)$ & & & $0 / 20(0 \%)$ & & \\
$p$ value & 0.0444 & & & & & \\
\hline
\end{tabular}


infection rates. It is also known that aging is strongly associated with the development of chronic conditions such as hypertension, diabetes, and cardiovascular disease [22]. A hallmark feature of these chronic conditions is changes in the patient's innate and adaptive immune function [23]. This further exacerbates their ability to clear pathogens and resolve tissue damage due to age-related changes in immune cells such as monocytes and macrophages [21]. Nevertheless, we could not establish that age by itself, was associated with higher infectious outcomes.

When determining which factors could explain differences in post-splenectomy infections, patient sex was also explored. It was previously suggested that males are more susceptible to infection, sepsis, multiple organ failure and mortality after traumatic injury [24], in addition to the male gender being identified as an independent risk factor for postsurgical infections. More specifically, sex hormones such as estrogen have an immuno-protective effect by reducing the production of pro-inflammatory cytokines [24]. Current literature also suggests that aging is associated with changes in sex hormones which have a greater impact on postsurgical outcomes. Older males have a greater decrease in the total amount of $\mathrm{T}$ and $\mathrm{B}$ cells than older females [21]. Although menopaused women also experience a decrease in $\mathrm{T}$ and $\mathrm{B}$ cells, treatment with hormone replacement therapies which are largely estrogen-based has shown to improve immune function by increasing circulating $\mathrm{B}$ cells and balancing levels of pro-inflammatory cytokines [21]. However, we found no difference in sex distribution between those who suffered an infectious outcome and those who did not.

Interestingly, we found that only patients with relatively low-grade splenic injuries developed post-splenectomy infection. It is well known that splenic injury leads to splenosis [25] and speculated that more severe injuries have higher amounts of splenosis. If splenosis does occur, it is not a foregone conclusion that these splenic implants will develop into robust immunological organs. [26].

It is well known that operative trauma from an open laparotomy can lead to a certain degree of immunosuppresion [27]. Laparoscopy has been shown to attenuate this immunosuppresion [28]. Therefore, it may be likely that laparoscopic splenectomy may be associated with better immunological response that open splenectomy. Although laparoscopic splenectomy for non-trauma conditions is quite common, for trauma, it is not norm. However, the TraumaLap Study Group has shown that laparoscopy for splenic injury is useful in the diagnosis of associated injuries concomitant with the splenic injury and may be the definitive management of high-grade splenic injuries which have failed non-operative management, with the potential for fewer adverse post-operative events $[29,30]$. Certainly, trauma surgeons should consider laparoscopy as an operative option.
The limitations with this study are recognized among all retrospective chart reviews in that there is potential for incomplete or incorrect documentation which could have impacted the quality of data collected. Furthermore, the retrospective nature of the study makes temporal and causal relationships harder to deduce. This retrospective chart review was non-blinded which could in part cause subjective bias. To address this, data abstractors were monitored throughout the data collection process. Their work was reviewed by the designated research coordinators and finally by the data analyst. Our sample size is relatively small and only included patients who received care at a single hospital. A larger study population across multiple sites would allow for improved generalizability. Lastly, we had $25 \%$ of our patients lost to follow-up and, therefore, they may have been treated at another institution for an infection of which we were not aware.

\section{Conclusion}

In conclusion, this study evaluated the rates of infections between traumatic and non-traumatic splenectomy patients. The results showed subjects in the non-traumatic category experienced a higher rate of infectious outcomes. This included patients undergoing splenectomy for disease and with a distal pancreatectomy. Vaccinations did not seem to provide a protective effect.

Funding One of the authors, Vic Velanovich, receives funding from Integra Biosciences and Innocoll Pharmaceuticals, neither of which has any relationship with this research.

\section{Declarations}

Competing interests The authors declare no competing interests.

\section{References}

1. Katz SC, Pachter HL (2006) Indications for splenectomy. Am Surg 72:565-580

2. Carr JA, Shurafa M, Velanovich V (2002) Surgical indications in idiopathic splenomegaly. Arch Surg 137:64-68

3. Girard E, Abba J, Cristiano N et al (2016) Management of splenic and pancreatic trauma. J Visc Surg 153(4 Suppl):45-60

4. Soares KC, Pawlik TM (2019) Techniques of pancreatic resection for cancer. In: Yeo CJ (ed) Shackelford's Surgery of the Alimentary Tract, 8th edn. Elseiver, Amsterdam, pp 1181-1192

5. Almond BA, Diehl KM (2010) Splenectomy. In: Minter RM, Doherty GM (eds) Current Procedures: Surgery. McGraw-Hill Companies, Inc., New York

6. Matharoo GS, Afthinos JN, Gibbs KE (2014) Trends in splenectomy: where does laparoscopy stand? JSLS 18(4):e2014.00239 
7. Velanovich V, Shurafa MS (2001) Clinical and quality of life outcomes of laparoscopic and open splenectomy for haematological diseases. Eur J Surg 167:23-28

8. Lewis SM, Williams A, Eisenbarth SC (2019) Structure-function of the immune system in the spleen. Sci Immunol 4(33):eaan6085

9. Kristinsson SY, Gridley G, Hoover RN et al (2014) Long-term risks after splenectomy among 8,149 cancer-free American veterans: a cohort study with up to 27 years follow-up. Haematologica 99:392-398

10. Sinwar PD (2014) Overwhelming post-splenectomy infection syndrome-a review study. Int J Surg 12:1314-1316

11. Okabayashi T, Hanazaki K (2008) Overwhelming postsplenectomy infection syndrome in adults - a clinically preventable disease. World J Gastroenterol 14:176-179

12. Olthof DC, van der Vlies LH, Goslings JC (2017) Evidence-based management and controversies in blunt splenic trauma. Curr Trauma Rep 3:32-37

13. Velanovich V, Weaver M (2003) Partial splenectomy using a coupled saline-radiofrequency hemostatic device. Am J Surg 185:66-68

14. Partelli S, Cirocchi R, Randolph J et al (2016) A systematic review and meta-analysis of spleen-preservation or ligation of the splenic artery and vein. Surgeon 14:109-118

15. Pasternack MS. Prevention of infection in patients with impaired splenic function. Up to Date. www.uptodate.com. Lasted accessed August 14, 2021

16. Rodeghiero F, Ruggeri M (2012) Short- and long-term risks of splenectomy for benign haematological disorders: should we revisit the indications? Br J Haematol 158:16-29

17. Di Cataldo A, Puleo S, Li Destri G et al (1987) Splenic trauma and overwhelming postsplenectomy infection. Br J Surg 74:343-345

18. Ashorobi D, Fernandez R. Asplenia. www.ncbi.nlm.nih.gov/ books/NBK538171/. Lasted accessed, August 13, 2021

19. Hammerquist RJ, Messerschmidt KA, Pottebaum AA, Hellwig TR (2016) Vaccinations in asplenic adults. Am J Health Syst Pharm 73:e220-e228

20. Montecino-Rodriguez E, Berent-Maoz B, Dorshind K (2013) Causes, consequences, and reversal of immune system aging. J Clin Invest 123:958-965
21. GubbelsBupp MR, Potlur T, Fink AL, Klein SL (2018) The confluence of sex hormones and aging on immunity. Front Immunol 9:1269

22. Davis JW, Chung R, Juarez DT (2011) Prevalence of comorbid conditions with aging among patients with diabetes and cardiovascular disease. Hawaii Med J 70:209-213

23. Rubinow KB, Rubinow DR (2017) In immune defense: redefining the role of the immune system in chronic disease. Dialogues Clin Neurosci 19:19-26

24. Bosch F, Angele MK, Chaudry IH (2018) Gender differences in trauma, shock and sepsis. Mil Med Res 5:35

25. Fremont RD, Rice TW (2007) Splenosis: a review. South Med J 100:589-593

26. Connell NT, Brunner AM, Kerr CA, Schiffman FJ (2011) Splenosis and sepsis: the born-again spleen provides poor protection. Virulence 2:4-11

27. Choilean NN, Redmond P (2006) Cell response to surgery. Arch Surg 141:1132-1140

28. Novitsky YW, Litwin DEM, Callery MP (2004) The net immunologic advantage of laparoscopic surgery. Surg Laparosc 18:1411-1419

29. Birindelli A, Martin M, Khan M et al (2021) Laparoscopic splenectomy as a definitive management option for high-grade trauma splenic injury when non operative management is not feasible or failed: a 5-year experience from a level one trauma center with minimally invasive surgery expertise. Updates Surg 73:1515-1531

30. Di Saverio B, Birindelli A, Podda M et al (2019) Trauma laparoscopy and the six w's: why, where, who, when, what and how. J Trauma Acute Care Surg 86:344-367

Publisher's note Springer Nature remains neutral with regard to jurisdictional claims in published maps and institutional affiliations. 